\title{
ON THE CRITICAL DEGREE OF DIFFERENTIABILITY OF A COMPLEX PLANAR CURVE
}

\author{
BY \\ JOSEPH BECKER ${ }^{1}$
}

\begin{abstract}
An example of a pair of complex analytic curves in $\mathbf{C}^{2}$ is given which have the same characteristic pairs but which do not have the same critical degree of differentiability.
\end{abstract}

In the theory of planar curves, the characteristic pairs play an important role. From them one can compute the multiplicity sequence, length of the conductor, the fundamental group of the complement, the Milnor number, etc. It has long been desired to find an analytic invariant of complex analytic planar curves which cannot be computed from the characteristic pairs. One such invariant [2], [20] is the length of $\theta / J$, where $J=\left(f_{x}, f_{y}\right)$ is the ideal in $\left.\mathbf{C}[x, y]\right]$ generated by the partial derivatives of the defining equation of the curve. I give here a different invariant using differentiable functions.

Let $V$ be the germ of a complex analytic set in $\mathbf{C}^{n}, p \in V$, and $\hat{\theta}_{p}(V), \tilde{\mathcal{\Theta}}_{p}(V)$, and $C_{p}^{k}(V)$ the germs of holomorphic, weakly holomorphic, and $k$ times continuously differentiable functions on $V$. By [11], there exists $k$ such that $C_{p}^{k}(V) \cap$ $\tilde{\theta}_{p}(V)=\hat{\theta}_{p}(V)$, that is, every weakly holomorphic function on $V$ which has an extension to the ambient space in a neighborhood of $p$ which is $k$ times continuously differentiable has an extension which is holomorphic. Let $\kappa$ be the minimal such $k$. It is clear that $\kappa$ is preserved by analytic change of coordinates. For $V$ irreducible at $p, V$ is regular if and only if $\kappa=0$.

In $\S \S 1-5$, we investigate the properties of $\kappa$ for irreducible curves in $\mathbf{C}^{2}$. In $\$ \S 1$ and 3 we consider curves with just one characteristic pair $(p, q)$ and recall [3] $q-1 \leqslant \kappa \leqslant[p(q-2) / q]+1$, that the maximal value of $\kappa$ is attained for the curve $Z^{p}=W^{q}$, and that this value may also be attained for curves with char pair $(p, q)$ which are not analytically equivalent to $Z^{p}=W^{q}$ (in contrast with $l(\theta / J)$, where the maximum is attained only at the special curve [20]). In $\$ \S 3$ and 4 we give an example of two curves with the same characteristic pair but different $\kappa$. In particular, for $t \rightarrow\left(t^{4}, t^{9}\right), \kappa=5$, but for $t \rightarrow\left(t^{4}, t^{9}+t^{10}\right), \kappa=3$. In general it seems difficult to characterize $\kappa$ in terms of any known invariant of the curve. However for low values of $\kappa$, this is quite easy: $\kappa>2$ if and only if the multiplicity of $V$ is $\geqslant 3$.

Received by the editors July 11, 1980 and, in revised form, November 19, 1980.

1980 Mathematics Subject Classification. Primary 32K15; Secondary 32B10, 14 H20.

Key words and phrases. Complex analytic curve, characteristic pairs, differentiable function, holomorphic, weakly holomorphic.

'This research was partially supported by NSF Grant No. MCS 77-00967. 
Associated to any Puiseux expansion of a complex curve $W$ is a monomial space curve $V$ such that $W$ is the generic projection of $V$, e.g. to $\left(t^{q}, t^{p}+c t^{r}\right), q<p<r$, we have the corresponding curve $\left(t^{q}, t^{p}, t^{r}\right)$. It is natural to try to relate the critical degree $\kappa(V)$ and $\kappa(\pi(V))$, where $\pi$ is a generic projection. (There is a relationship [14] for the length of jacobian ideal, e.g. this length is minimized at the monomial curve.) In $\S 6$ we will show that one can derive a relation between $\kappa(V)$ and $\kappa(\pi(V))$ under certain conditions, but that does not shed any light on the previously mentioned example $\left(t^{4}, t^{9}+t^{10}\right)$.

1. We begin by recalling some results from [3] without proof. The germ at the origin of any irreducible analytic curve $V$ in $\mathbf{C}^{2}$ can be normalized by a map $\theta(t)=\left(t^{q}, t^{p} u(t)\right)$, where $p>q, q$ does not divide $p$, and $u(t)$ is a holomorphic function with $u(0)=1$. If $V$ is singular at the origin, that is if $q \geqslant 2$, then $p$ and $q$ are invariant under biholomorphic change of coordinates. In this section we will restrict our attention to the case where $p$ and $q$ are relatively prime.

Let $N=[p(q-1) / q]$, where $[x]$ is the largest integer which is less than or equal to $x$. Notice that $p-2 \geqslant N \geqslant q-1$.

LEMMA. If $f \in \tilde{\mathcal{O}}(V)$ there are unique constants $b_{\mu \nu}$ such that

$$
f(z, w)-\sum_{\mu=1}^{q-1} \sum_{\nu=0}^{N-1} b_{\mu \nu} w^{\mu} z^{\nu-N} \in \mathcal{O}(V) .
$$

Furthermore $b_{\mu \nu}=0$ if $p \mu+q \nu<q N$.

COROLlaRy 1. $w^{q-1} / z$ is the most differentiable element of $\tilde{\mathcal{O}}-\hat{\theta}$.

We now recall some results on the extension of weakly holomorphic functions on the curve to differentiable functions on the ambient space. Let $C^{k}(V)$ denote the algebra of complex valued functions on $V$ which have a $k$ times continuously differentiable extension to the ambient space. Let $\theta(V)$ be the algebra of complex valued functions on $V$ which have a holomorphic extension to the ambient space. Let $\tilde{\theta}(V)$ be the algebra of continuous complex valued functions on $V$ which are holomorphic at each regular point. Let $\kappa$ be the smallest integer $k$ such that $C^{k}(V) \cap \tilde{\theta}(V)=\hat{\theta}(V)$.

Proposition 2. $C^{N}(V) \cap \tilde{\theta}(V)=\theta(V)$.

Proposition 3. $C^{k}(V) \cap \tilde{\mathcal{O}}(V)=\mathcal{O}(V)$, if $k>p(q-2) / q$. Hence

$$
\kappa \leqslant[p(q-2) / q]+1 \text {. }
$$

Proposition 4. There exists $f \in \tilde{\mathcal{O}}(V)-\mathcal{O}(V)$ with $f \in C^{q-2}(V)$.

Corollary 2. $q-1 \leqslant \kappa \leqslant[p(q-2) / q]+1$.

One may easily find $p$ and $q$ such that $q-1=[p(q-2) / q]+1$, for instance $p=q+1$. On the other hand for $p \gg q$, we have $q-1<[p(q-2) / q]+1$. For the curve $Z^{p}=W^{q}, \kappa=[p(q-2) / q]+1$, but for more general curves $\kappa$ might be any of the values allowed in Corollary 2 . In $\$ \S 2-4$, we will show that for the curve $t \rightarrow\left(t^{4}, t^{9}\right), \kappa=5$, but for the curve $t \rightarrow\left(t^{4}, t^{9}+t^{19}\right), \kappa=3$. 
2. It is easy to show that if $V$ and $V^{\prime}$ are two planar curves of multiplicity 2 and with the same characteristic pair $(p, 2)$, then $V$ and $V^{\prime}$ are analytically equivalent and hence have the same $\kappa=1$ (by Corollary 3). So one need only study curves of multiplicity $\geqslant 3$. We now show any such curve always has $C^{1}$ weakly holomorphic functions which are not holomorphic.

Proposition 5. If $V$ is a planar curve of multiplicity $q \geqslant 3$, then $\kappa \geqslant 2$.

Proof. We will give a weakly holomorphic function and its $C^{1}$ extension by the Whitney extension theorem. As usual, let

$$
\theta(t)=\left(t^{q}, t^{p} u(t)\right), \quad u(t)=\sum_{i=0}^{\infty} a_{i} t^{i}
$$

be the normalization of $V$.

We begin by defining a sequence of integers $\left(l_{i}\right)$ as follows: set $l_{0}=q$ and assuming $l_{1}, \ldots, l_{r}$ chosen, let $l_{r+1}$ be the smallest integer $l>l_{r}$ such that $a_{l} \neq 0$ and $l$ is not of the form $l=\sum_{i=1}^{r} \alpha_{i} l_{i}$ for any nonnegative integers $\alpha_{i}$. We continue until G.C.D. $\left(l_{1}, \ldots, l_{k}\right)=1$. Since $\theta$ is a homeomorphism this process is finite. We claim there is an integer $c>\max \left\{l_{i}\right\}$ which is not of the form $\Sigma_{i-1}^{k} \alpha_{i} l_{i}, \alpha_{i} \in \mathrm{N}$. Let $g=$ G.C.D. of $l_{1}, \ldots, l_{k-1}$; note $g \geqslant 2$. Let $\left(d_{j}\right)$ be the set of integers of the form $d_{j}=\sum_{i=1}^{k-1} \alpha_{i, j} l_{i}, \alpha_{i, j} \in \mathbf{N}$, arranged in ascending order and let $m$ be the index such that $d_{m}<l_{k}<d_{m+1}$. Note that $d_{j+1}-d_{j} \geqslant g \geqslant 2$ for all $j$. If $l_{k} \neq d_{m+1}-1$, set $c=d_{m+1}-1$. Because $0<c-l_{k}<g, c$ has the required property. If $l_{k}=d_{m+1}-$ 1 , set $c=d_{m+1}+1$. Then $c-l_{k}=2$ and each $l_{i} \geqslant 3$, so $c$ has the required property.

Then $t^{c}$ is weakly holomorphic on $V$, but not holomorphic since $c$ is not in the semigroup generated by the $l_{i}$. To give a $C^{1}$ extension of $h(t)=t^{c}$ we let $h_{\bar{z}}=h_{\bar{w}}=h_{z} \equiv 0$, and $h_{w}=c t^{c-p} /\left(p u+t u^{\prime}\right)$. This data satisfies the chain rules $c t^{c-1}=q t^{q-1} h_{z}+\partial_{t}\left(t^{p} u\right) h_{w}$, and $0=q t^{q-1} h_{\bar{z}}+\partial_{t}(\bar{t} \bar{u} \bar{u}) h_{\bar{w}}$, so we need only check the limit at the origin. We need to show that

$$
\lim _{\substack{s, t \rightarrow 0 \\ s \neq t}} \frac{\left|\left(s^{c}-t^{c}\right)-\left(s^{p} u(s)-t^{p} u(t)\right) h_{w}(t)\right|}{\left|s^{q}-t^{q}\right|+\left|s^{p} u(s)-t^{p} u(t)\right|}=0 .
$$

Since $h_{w}(0)=0$, it is clear we may replace the top by just $\left|s^{c}-t^{c}\right|$. To prove this limit is zero it is sufficient to prove the limit is zero along every sequence $\left(s_{j}, t_{j}\right) \rightarrow(0,0)$. By passing to a subsequence, we may assume that either $s_{j} / t_{j}$ or its inverse approaches a complex number of norm $\leqslant 1$. Suppose $s_{j} / t_{j} \rightarrow \lambda$. Let

$$
\theta_{2}(s, t)=\left(s^{p} u(s)-t^{p} u(t)\right) /(s-t)
$$

and for $l>1$, let

$$
g_{l}(s, t)=\frac{\left(s^{l}-t^{l}\right)}{(s-t)}=\sum_{i=0}^{l} s^{i} t^{l-i-1} .
$$

In order to show the limit is zero, it suffices to show one of the following:

$$
\lim _{j \rightarrow \infty} \frac{g_{c}\left(s_{j}, t_{j}\right)}{g_{q}\left(s_{j}, t_{j}\right)}=0
$$




$$
\lim _{j \rightarrow \infty} \frac{g_{c}\left(s_{j}, t_{j}\right)}{\theta_{2}\left(s_{j}, t_{j}\right)}=0
$$

This requires a calculation. Because G.C.D. $\left(l_{i}\right)=1$, we have $g_{l_{1}}(1, \lambda) \neq 0$ for some $i$. Let $l_{h}$ be the smallest $l_{i}$ for which this is true. If $h=0$, then we see (A) holds:

$$
\begin{aligned}
\frac{g_{c}\left(s_{j}, t_{j}\right)}{g_{q}\left(s_{j}, t_{j}\right)} & =\frac{s_{j}-t_{j}}{s_{j}-t_{j}} \frac{s_{j}^{c-1}+\cdots+t_{j}^{c-1}}{s_{j}^{q-1}+\cdots+t_{j}^{q-1}} \\
& =\frac{s_{j}^{c-q} \sum_{i=0}^{q-1}\left(s_{j} / t_{j}\right)^{i}+\sum_{i=1}^{c-q} s_{j}^{c-q-i} t_{j}^{i-1}}{\sum_{i=0}^{q-1}\left(s_{j} / t_{j}\right)^{i}} \\
& \rightarrow\left[0 \cdot \sum_{i=0}^{q-1} \lambda^{i}+0\right] / \sum_{i=0}^{q-1} \lambda^{i}=0 .
\end{aligned}
$$

In any case, since $l_{h}<c$, the above argument shows that

$$
\lim _{j \rightarrow \infty} g_{c}\left(s_{j}, t_{j}\right) / g_{l_{h}}\left(s_{j}, t_{j}\right)=0
$$

and, hence, to prove (A) or (B), it suffices to show either $\left(\mathrm{A}^{\prime}\right)$ or $\left(\mathrm{B}^{\prime}\right)$.

(A') $\lim _{j \rightarrow \infty} g_{l_{h}}\left(s_{j}, t_{j}\right) / g_{q}\left(s_{j}, t_{j}\right)$ is bounded.

$\left(\mathrm{B}^{\prime}\right) \lim _{j \rightarrow \infty} g_{l_{h}}\left(s_{j}, t_{j}\right) / \theta_{2}\left(s_{j}, t_{j}\right)$ is bounded.

Let

$$
\begin{array}{cr}
s_{j}=t_{j} \lambda_{j}=\lambda t_{j}+t_{j}\left(\lambda_{j}-\lambda\right)=t_{j}\left(\lambda+\varepsilon_{j}\right), & \varepsilon_{j} \rightarrow 0, \quad \lambda \neq 0 . \\
s_{j}^{l}-t_{j}^{l}=t_{j}^{l}\left(\lambda^{l}-1\right)+t_{j}^{l}\left(l \lambda^{l-1} \varepsilon_{j}\right) u_{j l}, \quad u_{j l} \rightarrow 1 \quad \text { as } j \rightarrow \infty .
\end{array}
$$

If $\lambda^{q}=1$ and $\left(\mathrm{A}^{\prime}\right)$ fails, then $t_{j}^{l_{h}-q} / \varepsilon_{j} \rightarrow \infty$. Hence $\varepsilon_{j} t_{j}^{l-l_{h}} \rightarrow 0$ for each $l>q$. But

$$
\theta_{2}\left(s_{j}, t_{j}\right)=\varepsilon_{j} \sum_{l_{1}<k<l_{h}} a_{k} k \lambda^{k-1} u_{j k} t_{j}^{k}+t_{j}^{h^{h}} S_{j}
$$

$S_{j} \rightarrow S, S \neq 0$. Hence $\theta_{2}\left(s_{j}, t_{j}\right)=O\left(t_{j}^{h}\right)$ and $\left(\mathrm{B}^{\prime}\right)$ holds.

REMARK. Conversely if $V$ is a planar curve with $\kappa \geqslant 2$, then the multiplicity $q \geqslant 3$. This follows from Corollary 2 .

3. Now let us restrict our attention to the curve $V$ in $\mathbf{C}^{2}, Z^{p}=W^{q}, p>q$, $(p, q)=1$. Let $h_{\mu \nu}=w^{\mu} / z^{\nu}$ and $\sigma=p \mu-q \nu$; then $h_{\mu \nu}(\theta(t))=t^{\sigma}$. We recall from [3]:

THEOREM 1. $h_{\mu \nu} \in C^{m}(V)$ where

$$
m= \begin{cases}{[\sigma / p],} & {[\sigma / p]<q-\mu,} \\ q-\mu+[(\sigma-p(q-\mu)) / q], & {[\sigma / p]>q-\mu .}\end{cases}
$$

THEOREM 2. $h_{\mu \nu} \notin C^{m+1}(V)$.

COROLlaRY 3. For the curve $Z^{p}=W^{q}, \kappa=[p(q-2) / q]+1$.

Proof. Apply Theorems 1 and 2 to $w^{q-1} / z$.

Now combining the statements of Corollaries 2 and 3, we see that over the class of curves with one char pair $(p, q), \kappa$ is maximized at the curve $Z^{p}=W^{q}$. However 
it is not strictly maximized: An elementary power series calculation shows that $t \rightarrow\left(t^{4}, t^{5}\right)$ and $t \rightarrow\left(t^{4}, t^{5}+t^{6}\right)$ are not analytically equivalent. Corollary 2 shows that for each of these curves $\kappa=3$. So analytically inequivalent curves with the same characteristic pairs might have the same $\kappa$. (More generally for any $q>1$, $t \rightarrow\left(t^{q}, t^{q+1}\right)$ and $t \rightarrow\left(t^{q}, t^{q+1} u(t)\right)$, both have $\kappa=q-1$.) In order to find an example of curves in $\mathbf{C}^{2}$, both with char pair $(p, q)$ but with different $\kappa$ we must pick $q-1<[p(q-2) / q]+1$. In particular, $t \rightarrow\left(t^{4}, t^{9}\right)$ has $\kappa=5$ and $t \rightarrow\left(t^{4}, t^{9}\right.$ $\left.+t^{10}\right)$ has $\kappa=3$.

4. Special examples. We will now show that for the curve $t \rightarrow\left(t^{4}, t^{9}+t^{19}\right)$, $\kappa \leqslant 4$. It suffices to show that $t^{23} \notin C^{4}(V)$.

Suppose $t^{23}$ has a $C^{4}$ extension $F$ to $C^{2}$; then there exist continuous $F_{\alpha}=D^{\alpha} F$ defined on $\mathbf{C}^{2}$ so that

$$
F(x)=\sum_{|\alpha|<4} \frac{(x-y)^{\alpha}}{\alpha !} F_{\alpha}(y)=o\left(|x-y|^{4}\right) .
$$

Composing with $x=\theta(s), y=\theta(t)$ and letting $f_{\alpha}(s)=F_{\alpha}(\theta(s)), f_{\alpha}(t)=F_{\alpha}(\theta(t))$, we have that for each $\alpha$,

$$
f_{\alpha}(s)-\sum_{|\alpha+\beta|<4} \frac{(\theta(s)-\theta(t))^{\beta}}{\beta !} f_{\alpha+\beta}(t)=o\left(|\theta(s)-\theta(t)|^{4-|\alpha|}\right) .
$$

In particular,

$$
f(s)-\sum_{|\beta|<3} \frac{(\theta(s)-\theta(t))^{\beta}}{\beta !} f_{\beta}(t)=o\left(\left|s^{4}-t^{4}\right|^{3}+\left|s^{9}+s^{10}-t^{9}-t^{10}\right|^{3}\right) .
$$

Substituting $s=\omega t, \omega^{2} t, \omega^{3} t$, where $\omega$ is a primitive 4 th root of unity yields

$$
\left(\omega^{l \sigma}-1\right) t^{\sigma}-\sum_{|\beta|<3} \frac{\left(\theta\left(\omega^{l} t\right)-\theta(t)\right)^{\beta}}{\beta !} f_{\beta}(t)=o\left(t^{27}\right) .
$$

First let us assume the Taylor series of $f$ at the origin is zero; then by the Taylor remainder theorem,

$$
f_{\alpha}(x)-T_{0}^{4-|\alpha|} f_{\alpha}(x)=o\left(|x|^{4-|\alpha|}\right)=o\left(t^{16-4|\alpha|}\right) .
$$

In particular for any $|\alpha|=2, f_{\alpha}(t)=o\left(t^{8}\right)$ and for any $|\alpha|=3, f_{\alpha}(t)=o\left(t^{4}\right)$. Hence for

$$
\begin{array}{ll}
|\beta|=3, & \left(\theta\left(\omega^{l} t\right)-\theta(t)\right)^{\beta} f_{\beta}(t)=O\left(t^{27}\right) o\left(t^{4}\right)=o\left(t^{31}\right) \\
|\beta|=2, & \left(\theta\left(\omega^{l} t\right)-\theta(t)\right)^{\beta} f_{\beta}(t)=O\left(t^{18}\right) O\left(t^{8}\right)=o\left(t^{25.5}\right) .
\end{array}
$$

Substituting this into (E4) and considering just the first order terms:

$$
\begin{aligned}
\left(\omega^{l o}-1\right) t^{23}-\left[\left(\omega^{9 l}-1\right) t^{9}+\left(\omega^{10 l}-1\right) t^{10}\right] f_{3}(t) \\
-\left[\left(\bar{\omega}^{9 l}-1\right) t^{9}+\left(\bar{\omega}^{10 l}-1\right) t^{10}\right] f_{4}(t)=o\left(t^{25.5}\right) .
\end{aligned}
$$

Letting $l=2,3$ as above:

$$
\begin{gathered}
\left(\begin{array}{c}
\omega^{2}-1 \\
\omega-1
\end{array}\right) t^{14}-\left(\begin{array}{cc}
\omega^{2}-1 & \omega^{2}-1 \\
\omega^{3}-1+t\left(\omega^{2}-1\right) & \omega-1+\bar{t}\left(\omega^{2}-1\right)
\end{array}\right)\left[\begin{array}{c}
f_{3}(t) \\
(\bar{t} / t)^{9} f_{4}(t)
\end{array}\right) \\
=o\left(t^{16.5}\right) .
\end{gathered}
$$


This matrix is nonsingular as

$$
\left|\begin{array}{cc}
\omega^{2}-1 & \omega^{2}-1 \\
\omega^{3}-1 & \omega-1
\end{array}\right|=-\omega\left(\omega^{2}-1\right) \neq 0
$$

Multiplying the equation by the inverse of the matrix:

$$
\begin{aligned}
o\left(t^{16.5}\right)= & \frac{\left(\omega^{2}-1\right)^{-2}}{-\omega+\bar{t}-t}\left[\begin{array}{cc}
\omega-1+\bar{t}\left(\omega^{2}-1\right) & 1-\omega^{2} \\
1-\omega^{3}+t\left(1-\omega^{2}\right) & \omega^{2}-1
\end{array}\right]\left[\begin{array}{c}
\omega^{2}-1 \\
\omega-1
\end{array}\right] t^{14} \\
& -\left[\begin{array}{c}
f_{3}(t) \\
(\bar{t} / t)^{9} f_{4}(t)
\end{array}\right] \\
= & {\left[\begin{array}{c}
t^{14} \\
\omega-\bar{t}+t
\end{array}\right]\left[\begin{array}{c}
-\bar{t} \\
\omega+t
\end{array}\right]-\left[\begin{array}{c}
f_{3}(t) \\
(\bar{t} / t)^{9} f_{4}(t)
\end{array}\right] . }
\end{aligned}
$$

Hence

$$
f_{3}(t)=t^{14}\left(C_{1} \bar{t}^{-}+C_{2} t^{2}+C_{3} t \bar{t}\right)+\text { higher order terms } H_{3},
$$

where $C_{1} \neq 0, C_{2} \neq 0, C_{3} \neq 0$ are constants, and $H_{3}(t)=o\left(t^{16}\right)$.

$$
f_{4}(t)=t^{23} / \bar{t}^{9}\left(C_{0}^{\prime}+C_{1}^{\prime} \bar{t}+C_{2}^{\prime} t^{2}+C_{3}^{\prime} t \bar{t}\right)+H_{4},
$$

where $C_{0}^{\prime}, C_{1}^{\prime}, C_{2}^{\prime}, C_{3}^{\prime}$ are nonzero constants and $H(t)=o\left(t^{16}\right)$.

Now consider the second order Taylor series of $f_{3}$ :

$$
\begin{aligned}
f_{3}(s)-f_{3}(t)-[ & \left(s^{4}-t^{4}\right) g_{1}(t)+\overline{\left(s^{4}-t^{4}\right)} g_{2}(t) \\
& \left.+\left(s^{9}+s^{10}-t^{9}-t^{10}\right) g_{3}(t)+\overline{\left(s^{9}+s^{10}-t^{9}-t^{10}\right)} g_{4}(t)\right] \\
& +2 \text { nd order terms } \\
= & o\left(|\theta(s)-\theta(t)|^{2}\right) .
\end{aligned}
$$

Restricting to the lines $s=\omega^{2} t, \omega^{3} t$, we have

$$
\left(\begin{array}{c}
\omega^{2}-1 \\
\omega^{3}-1
\end{array}\right) t^{5} \bar{t}-\left(\begin{array}{cc}
\omega^{2}-1 & \omega^{2}-1 \\
\omega^{3}-1+t\left(\omega^{2}-1\right) & \omega-1+\bar{t}\left(\omega^{2}-1\right)
\end{array}\right)\left(\begin{array}{c}
g_{3}(t) \\
(\bar{t} / t)^{9} g_{4}(t)
\end{array}\right)=o\left(t^{7}\right) \text {. }
$$

Multiplying through by the inverse of the matrix:

$$
\frac{t^{5} \bar{t}}{\omega-\bar{t}+t}\left[\begin{array}{c}
-\omega+\bar{t} \\
t
\end{array}\right]-\left[\begin{array}{c}
g_{3}(t) \\
(\bar{t} / t)^{9} g_{4}(t)
\end{array}\right]=o\left(t^{7}\right) \text {. }
$$

Hence

$$
\begin{aligned}
g_{3}(t)-C t^{5} t & =o\left(t^{6}\right), & C \neq 0, \\
g_{4}(t)-C^{\prime} t^{15} / t^{8} & =o\left(t^{7}\right), & C^{\prime} \neq 0 .
\end{aligned}
$$

But $g_{3}$ and $g_{4}$ are both twice differentiable. For $i=3,4, g_{i}-T_{0}^{2} g_{i}=o\left(t^{8}\right)$, so

$$
t^{5} t-T_{0}^{2} g_{3}=o\left(t^{6}\right) \text { and } t^{15} / t^{8}-T_{0}^{2} g_{4}=o\left(t^{7}\right) \text {. }
$$


Since the second order Taylor series is all monomials in $t^{4}, t^{4}, t^{9}+t^{10}$, and $\bar{t}^{9}+\bar{t}^{10}$, it is easily seen that neither $t^{5} \bar{t}$ nor $t^{14} / \bar{t}^{8}$ can be cancelled by the Taylor series. Hence we have a contradiction.

Alternately we could apply the preceding argument to $g_{4}$, i.e., $g_{4}(\omega t)-g_{4}(t)=$ $O\left((\omega-1) t^{9}\right)$ by a first order Taylor estimate, but $g_{4}(\omega t)-g_{4}(t)=\left(\omega^{3}-1\right) t^{7}+$ $o\left(t^{7}\right)$ by the previous results (E6). This gives a contradiction.

In general, we do not have the Taylor series of $f$ is zero, so we replace $f$ by $h=f-P f$, where $P f$ is the partial Taylor series of $f$ about the origin consisting of all the terms from $f_{w z}, f_{w \bar{z}}, f_{\bar{w} z}, f_{\bar{w} \bar{z}} ; f_{w w}, f_{w \bar{w}}, f_{\bar{w} \bar{w}} ; f_{w z z}, f_{w z \bar{z}}, f_{w \bar{z} \bar{z}}, f_{\bar{w} z z}, f_{\bar{w} \bar{z} \bar{z}}, f_{\bar{w} \bar{z} \bar{z}} ; f_{w w z}, f_{w w \bar{z}}$, $f_{\bar{w} \bar{w} z}, f_{\bar{w} \bar{z} \bar{z}}, f_{w \bar{w} z}, f_{w \bar{w} \bar{z}} ; f_{w w w}, f_{w w \bar{w}}, f_{w \bar{w} \bar{w}}, f_{\bar{w} \bar{w} \bar{w}}$. Let $I$ be the set of multi-indices $\alpha$ associated to the derivatives in the list. Note that $f-P f$ does not necessarily have any $t^{23}$ term because if $f_{z w w}(0) \neq 0$, it gives rise to a $t^{23}$ term which might cancel out $f$. However $h(t)$ is a polynomial in $t$ and $\bar{t}$. Note

(a) $\operatorname{ord}_{0} h \leqslant 23$;

(b) for $\alpha \in I, h_{\alpha}(0)=0$;

(c) for $|\alpha|=2, h_{\alpha}(t)=o\left(t^{7.5}\right)$;

(d) for all $\alpha$,

$$
h_{\alpha}(s)-\sum_{|\beta|<4-|\alpha|} \frac{(\theta(s)-\theta(t))^{\beta}}{\beta !} h_{\alpha+\beta}(t)=o\left(|\theta(s)-\theta(t)|^{4-|\alpha|}\right) ;
$$

(e) the initial terms of $h_{\alpha}, \alpha \in I$, are all distinct, and $\left\{\operatorname{rot}\left(h_{\alpha}\right)\right\}_{\alpha \in I}$ are all distinct. For a polynomial $h$ in $t$ and $\bar{t}$, let ord $h$ denote its order as a power series in $t$ and $\bar{t}$. Let int $h$ be the initial term of $h$ as a power series in $t$ and $\bar{t}$. Let rot $h$ be the rotation of $h$, that is, the finite set of integers $\{i-j\}$, one for each monomial $t^{i} \bar{t}^{j}$ with nonzero coefficient in the initial term of $h$, i.e. $\operatorname{rot}\left(t^{i} t^{j}\right)=i-j$.

Next consider the case where $P f$ has only one monomial $t^{i} t^{j}$ in its initial term. Let $r=\operatorname{rot}(h) \bmod 4,0 \leqslant r \leqslant 3$. Now the argument proceeds as before. We have

$$
\begin{gathered}
\left(\begin{array}{c}
\omega^{2 r}-1 \\
\omega^{3 r}-1
\end{array}\right) \frac{t^{i} t^{j}}{t^{9}}-\left(\begin{array}{cc}
\omega^{2}-1 & \omega^{2}-1 \\
\omega^{3}-1+t\left(\omega^{2}-1\right) & \omega-1+\bar{t}\left(\omega^{2}-1\right)
\end{array}\right)\left(\begin{array}{c}
h_{3}(t) \\
(\bar{t} / t)^{9} h_{4}(t)
\end{array}\right) \\
=o\left(t^{i+j-9+1 / 2}\right) .
\end{gathered}
$$

Inverting the matrix, we get

$$
o\left(t^{i+j-8.5}\right)=\frac{1}{\omega-\bar{t}+t} A \cdot t^{i-9 \bar{t}^{j}}-\left[\begin{array}{c}
h_{3}(t) \\
(\bar{t} / t)^{9} h_{4}(t)
\end{array}\right]
$$

where

$$
A=\left\{\begin{array}{cccc}
r=1 & r=3 & r=2 & r=0 \\
{\left[\begin{array}{c}
-\omega+\bar{t} \\
t
\end{array}\right]} & {\left[\begin{array}{c}
-\bar{t} \\
\omega+t
\end{array}\right]} & {\left[\begin{array}{l}
-1 \\
-1
\end{array}\right]} & {\left[\begin{array}{l}
0 \\
0
\end{array}\right] .}
\end{array}\right.
$$

Previously we continued to work with $h_{3}$-now depending on $r$, we work with either $h_{3}$ or $h_{4}$-whichever has odd rotation. By repeating the same argument we again arrive at a contradiction. 


\begin{tabular}{|l|c|c|c|}
\multicolumn{1}{c}{} & rot $h$ & rot $h_{3}$ & rot $h_{4}$ \\
\hline$f_{\bar{w} z}, f_{\bar{w} \bar{z}}, f_{\bar{w} z z}, f_{\bar{w} z \bar{z}}, f_{\bar{w} \bar{z} \bar{z}}$ & 3 & 1 & 2 \\
\hline$f_{w w}, f_{\bar{w} \bar{w}}, f_{w w z}, f_{w w \bar{z}}, f_{\bar{w} \bar{w} \bar{z}}, f_{\bar{w} \bar{w} \bar{z}}$ & 2 & 1 & 1 \\
\hline$f_{w z}, f_{w \bar{z}}, f_{w z z}, f_{w \bar{z}}, f_{w \bar{z} \bar{z}}$ & 1 & 0 & 0 \\
\hline$f_{w \bar{w}}, f_{w \bar{w} z}, f_{w \bar{w} \bar{z}}$ & 0 & - & - \\
\hline$f_{w w w}, f_{w w \bar{w}}, f_{w \bar{w} \bar{w}}, f_{\bar{w} \bar{w} \bar{w}}$ & 3 & 1 & 2 \\
\hline
\end{tabular}

The last row gives rise to terms of order $>23$ so it can be forgotten in the calculation. The second to last row has a slight extra problem-for instance

$$
f_{w \bar{w}}=t^{9} t^{9}+t^{9} t^{10}+t^{10} t^{9}+t^{10} t^{10},
$$

$t^{9} t^{9}$ rotates itself away, but $t^{9} t^{10}$ and $t^{10} t^{9}$ have rotation 3 and $1 \bmod 4$, respectively, and the corresponding $\left(h_{3}, h_{4}\right)$ have rotation $(1,2)$ and $(0,1)$, respectively. So we may work with either $h_{3}$ or $h_{4}$.

The general case where int $P F$ has several monomials proceeds exactly as the above argument. One simply introduces enough notation to keep track of the various coefficients. The details are left to the reader.

5. Examples continued. We now improve the results in $\$ 4$ to show that $t^{23} \notin$ $C^{3}(V)$ and, hence, by the remark of $\S 3$, that $\kappa=3$.

We assume $t^{23} \in C^{3}(V)$ and argue to a contradiction. Let

$$
\begin{gathered}
f_{3}=f_{w}(t), \quad f_{4}=(\bar{t} / t)^{9} f_{\bar{w}}(t), \quad g_{1}=\frac{1}{2} f_{w w}(t), \\
g_{2}=\frac{1}{2}(\bar{t} / t)^{18} f_{\bar{w} \bar{w}}(t), \quad g_{3}=(\bar{t} / t)^{9} f_{w \bar{w}}(t) .
\end{gathered}
$$

We let $\omega=i$ be our primitive 4th root of unity. Note that by a first order Taylor series estimate restricted to the lines $s=i^{l} t$ :

$$
g_{j}\left(i^{l} t\right)-g_{j}(t)=O\left(t^{9}\right) \quad \text { for } j=1,2,3, l=1,2,3 .
$$

Next, considering a 3rd order Taylor estimate of $f$ restricted to the lines $s=t$, $-t,-i t$ and with $t$ replaced by $+i t$, throwing away the third order terms we have

$$
\begin{gathered}
t^{14}\left[\begin{array}{c}
1+i \\
-1+i \\
-2 i
\end{array}\right]+\left[\begin{array}{cc}
1-i+2 t & 1+i+2 \bar{t} \\
-1-i+2 t & -1+i+2 \bar{t} \\
-2 i & 2 i
\end{array}\right]\left[\begin{array}{l}
f_{3}(i t) \\
f_{4}(i t)
\end{array}\right] \\
+\left[\begin{array}{ccc}
-2 i & 2 i & 2 \\
2 i & -2 i & 2 \\
-4 & -4 & +4
\end{array}\right]\left[\begin{array}{l}
g_{1}(i t) \\
g_{2}(i t) \\
g_{3}(i t)
\end{array}\right] t^{9}=O\left(t^{15}\right),
\end{gathered}
$$

where the last matrix has been written modulo the ideal generated by $t$ and $\bar{t}$. Performing row operations, this becomes

$$
t^{14}\left[\begin{array}{c}
2 \\
0 \\
+1
\end{array}\right]+\left[\begin{array}{cc}
0 & 2 \\
0 & 0 \\
1 & -1
\end{array}\right]\left[\begin{array}{l}
f_{3} \\
f_{4}
\end{array}\right]+\left[\begin{array}{ccc}
2 & 4 i+2 & -2 i \\
0 & 4 & 0 \\
-2 i & -2 i & +2 i
\end{array}\right]\left[\begin{array}{l}
g_{1} \\
g_{2} \\
g_{3}
\end{array}\right] t^{9}=O\left(t^{15}\right)
$$


where both the first and third matrices are written $\bmod (t, \bar{t})$. From the second row, $g_{2}(i t)=O\left(t^{6}\right)$. Hence we have

$$
g_{2}(t)=O\left(t^{6}\right)
$$

Repeating this Taylor estimate with $s=t,-t$, it and $t$ replaced with $-i t$, we have

$$
\begin{array}{r}
t^{14}\left[\begin{array}{c}
1-i \\
-2 i \\
-1-i
\end{array}\right]+\left[\begin{array}{cc}
1+i+2 t & 1-i+2 \bar{t} \\
2 i & -2 i \\
-1+i+2 t & -1-i+2 t
\end{array}\right]\left[\begin{array}{l}
f_{3}(-i t) \\
f_{4}(-i t)
\end{array}\right] \\
+\left[\begin{array}{ccc}
2 i & -2 i & 2 \\
-4 & -4 & 4 \\
-2 i & 2 i & +2
\end{array}\right]\left[\begin{array}{l}
g_{1}(-i t) \\
g_{2}(-i t) \\
g_{3}(-i t)
\end{array}\right] t^{9}=O\left(t^{15}\right),
\end{array}
$$

where the last matrix is written mod $(t, \bar{t})$. Performing row operations yields

$$
t^{14}\left[\begin{array}{l}
2 \\
1 \\
0
\end{array}\right]+\left[\begin{array}{cc}
0 & 2 \\
-1 & 1 \\
0 & 0
\end{array}\right]\left[\begin{array}{l}
f_{3} \\
f_{4}
\end{array}\right]+\left[\begin{array}{ccc}
2 & 4-4 i & 2 i \\
-2 i & -2 i & 2 i \\
2 & 4 & 0
\end{array}\right]\left[\begin{array}{l}
g_{1} \\
g_{2} \\
g_{3}
\end{array}\right] t^{9}=O\left(t^{15}\right)
$$

Hence $g_{1}(-i t)+g_{2}(-i t)=O\left(t^{6}\right)$. By (E8) and (E7), we have

$$
g_{1}(t)=O\left(t^{6}\right)
$$

Again restrict the Taylor estimate to $s=t$, it, $-i t$ with $t$ replaced by $-t$, giving

$$
\begin{aligned}
t^{14}\left[\begin{array}{c}
2 \\
1-i \\
1+i
\end{array}\right]+ & {\left[\begin{array}{cc}
2+2 t & 2+2 t \\
1+i & 1-i \\
1-i & 1+i
\end{array}\right]\left[\begin{array}{l}
f_{3}(-t) \\
f_{4}(-t)
\end{array}\right] } \\
& +\left[\begin{array}{ccc}
4 & 4 & 4 \\
2 i & -2 i & 2 \\
-2 i & 2 i & 2
\end{array}\right]\left[\begin{array}{l}
g_{1}(-t) \\
g_{2}(-t) \\
g_{3}(-t)
\end{array}\right] t^{9}=O\left(t^{15}\right)
\end{aligned}
$$

where the last matrix is written $\bmod (t, \bar{t})$. Performing row operations, we have

$$
t^{14}\left[\begin{array}{c}
2 \\
-4 \\
0
\end{array}\right]+\left[\begin{array}{cc}
2 i & 2 \\
0 & -4 \\
0 & 0
\end{array}\right]\left[\begin{array}{l}
f_{3} \\
f_{4}
\end{array}\right]+\left[\begin{array}{ccc}
2 i-2 & 2-2 i & 2+2 i \\
8 i & -8 & 0 \\
2 i-1 & -1 & 1
\end{array}\right]\left[\begin{array}{l}
g_{1} \\
g_{2} \\
g_{3}
\end{array}\right]=O\left(t^{15}\right)
$$

Hence

$$
(2 i-1) g_{1}(-t)-g_{2}(-t)+g_{3}(-t)=O\left(t^{6}\right) .
$$

By (E7)-(E9), we have

$$
g_{3}(t)=O\left(t^{6}\right)
$$

By (E8)-(E10), we can find a $3 \times 3$ matrix $A$ with entries being polynomials in $t$ and $\bar{t}$ such that $A \bmod (t, \bar{t})=I$ and

$$
A\left(\begin{array}{l}
g_{1} \\
g_{2} \\
g_{3}
\end{array}\right)=O\left(t^{6}\right)
$$


Since $A$ has entries in $\mathbf{C}\{t, \bar{t}\}$, the local ring of convergent power series, $A$ has an inverse matrix. Multiplying by $A^{-1}$, we have

$$
\text { Each } g_{i}(t)=O\left(t^{6}\right)
$$

Finally restrict a 3rd order Taylor series of $f$ to the lines $s=i t,-t,-i t$ to give

$$
\begin{array}{r}
t^{14}\left[\begin{array}{c}
-i-1 \\
-2 \\
i-1
\end{array}\right]+\left[\begin{array}{cc}
i-1-2 t & -i-1-2 \bar{t} \\
-2 & -2 \\
-i-1-2 t & i-1-2 \bar{t}
\end{array}\right]\left[\begin{array}{l}
f_{3}(t) \\
f_{4}(t)
\end{array}\right] \\
+\left[\begin{array}{ccc}
-2 i & 2 i & 2 \\
4 & 4 & 4 \\
2 i & -2 i & 2
\end{array}\right]\left[\begin{array}{l}
g_{1}(t) \\
g_{2}(t) \\
g_{3}(t)
\end{array}\right] t^{9}=O\left(t^{15}\right) .
\end{array}
$$

By row operations, we get

$$
t^{14}\left[\begin{array}{c}
-2 \\
-1 \\
1
\end{array}\right]+\left[\begin{array}{cc}
0 & -2 i \\
-1 & -1 \\
0 & 0
\end{array}\right]\left[\begin{array}{l}
f_{3} \\
f_{4}
\end{array}\right]+t^{9}\left[\begin{array}{ccc}
-2 & 4 i-2 & 2 i \\
2 & 2 & 2 \\
1 & 1 & 0
\end{array}\right]\left[\begin{array}{l}
g_{1} \\
g_{2} \\
g_{3}
\end{array}\right]=O\left(t^{15}\right)
$$

Hence

$$
t^{14}+t^{9}\left[g_{1}(t)+g_{2}(t)\right]=O\left(t^{15}\right)
$$

By $(\mathrm{E} 11)$, we have $t^{14}=O\left(t^{15}\right)$. This is a contradiction.

6. It seems quite natural to ask what $\kappa$ is for the more general curve $\left(t^{4}, t^{9}+\right.$ $\left.c t^{10}\right), c$ a nonzero constant, i.e. the generic projection into $\mathbf{C}^{2}$ of the space curve $\left(t^{4}, t^{9}, t^{19}\right)$. By this point $\mathrm{I}$ am sure the reader would prefer that the details be suppressed. For $c \neq i,-i$, or $-1, \kappa=3$ as shown by the method of $\S 5$. However, for $c=i,-i$, or -1 , the calculation of $\S 5$ fails, but the method of $\$ 4$ shows that $\kappa<4$. In fact for these three values of $c, \kappa=4$. The proof of the inequality $\kappa>4$ will not be given here; the existence of the required extension of $t^{23}$ to $e^{2}$ can be demonstrated by using the Whitney extension theorem [15].

Also one may consider space curves and ask what $\kappa$ is for the curve $W$ in $\mathbf{C}^{3}$ given by $\left(t^{4}, t^{9}, t^{10}\right)$. Since [3, Theorem 5] $\kappa \leqslant$ the conductor number of the curve, i.e. the smallest integer $n$ such that ideal(sing $W)^{m} \tilde{\theta}(W) \subset \vartheta(W)$, we see that $\kappa \leqslant 4$. Since $t^{15} \in \tilde{\mathcal{O}}(W)-\mathcal{O}(W)$, and by Theorem $1, t^{15}$ on the curve $\left(t^{4}, t^{9}\right)$ has a $C^{1}$ extension, it follows that it has a $C^{1}$ extension on the curve $\left(t^{4}, t^{9}, t^{10}\right)$. Hence $\kappa \geqslant 2$ for the curve $\left(t^{4}, t^{9}, t^{10}\right)$. The author has not determined precisely what value $\kappa$ has for the curve $\left(t^{4}, t^{9}, t^{10}\right)$, only that $2 \leqslant \kappa \leqslant 4$.

It is natural to try to relate $\kappa$ of the space curve $\left(t^{4}, t^{9}, t^{10}\right)$ and $\kappa$ of its generic projection $\left(t^{4}, t^{9}+c t^{10}\right)$. With this goal in view we introduce the following definition of tangent space of an analytic variety with respect to $C^{k}$ functions.

Definition. Let $V$ be a germ of an analytic variety in $C^{n}$ and $I\left(V, C^{k}\right)$ the ideal of all germs of $C^{k}$ functions vanishing on $V$. Let

$$
T\left(V, C^{k}\right)=\left\{a \in \mathbf{C}^{n}=\mathbf{R}^{2 n} \mid 0=\sum_{i=1}^{n} a_{i} \frac{\partial f}{\partial z_{i}}+\bar{a}_{i} \frac{\partial f}{\partial \bar{z}_{i}} \text { for all } f \in I\left(V, C^{k}\right)\right\} .
$$


Clearly $T\left(V, C^{k}\right)$ is a real vector space but not a priori a complex vector space; $T\left(V, C^{k}\right)$ is easily seen to be the tangent space to the smallest $C^{k}$ manifold containing $V$. Clearly

$$
T\left(V, C^{k}\right) \subset T\left(V, C^{k+1}\right) \subset \ldots \subset T\left(V, C^{\infty}\right)=T(V, \mathcal{\theta}) .
$$

Also $T\left(V, C^{1}\right)$ is a complex vectorspace [6], and if $V$ is singular [4], then $\operatorname{dim} T\left(V, C^{1}\right)>\operatorname{dim} V$.

Proposition 6. Let $V$ be an irreducible complex analytic curve in $\mathbf{C}^{n}$, let $\Pi$ : $\mathbf{C}^{n} \rightarrow \mathbf{C}^{2}$ be a linear projection transversal to $T\left(V, C^{k}\right)$, i.e. $\Pi^{-1}(0) \cap T\left(V, C^{k}\right)=$ $\{0\}$, where $k=\kappa(\Pi(V))$. Then $\kappa(\Pi(V)) \geqslant \kappa(V)$.

Proof. It suffices to let $h \in \tilde{\mathcal{O}}(V) \cap C^{\mathrm{k}(\Pi(V))}$ and show $h \in \mathcal{O}(V)$. Now the projection $\Pi$ induces maps $\theta(\Pi(V)) \rightarrow \vartheta(V), \tilde{\theta}(\Pi(V)) \rightarrow \tilde{\theta}(V), C^{k}(\Pi(V)) \rightarrow$ $C^{k}(V)$. It is clear that $\tilde{\mathcal{O}}(\Pi(V))=\tilde{\mathcal{O}}(V)$ since both $V$ and $\Pi(V)$ are irreducible curves. Since $\Pi$ is transversal to $T\left(V, C^{k}\right)$, we also get an equality $C^{k}(\Pi(V))=$ $C^{k}(V)$. So

$$
\begin{aligned}
h \in \tilde{\mathcal{O}}(V) \cap C^{\kappa(\Pi(V))}(V) & \Rightarrow h \in \tilde{\mathcal{O}}(\Pi(V)) \cap C^{\kappa(\Pi(V))}(\Pi(V)) \\
& =\mathcal{O}(\Pi(V)) \Rightarrow h \in \mathcal{O}(V) .
\end{aligned}
$$

Unfortunately this result, while it does shed some light on the general question, does not help us with our particular example because in [5, Example 2], we showed that $T\left(\left(t^{4}, t^{9}, t^{10}\right), C^{2}\right)=\mathbf{C}^{3}$, so any projection $\Pi: \mathbf{C}^{3} \rightarrow \mathbf{C}^{2}$ cannot possibly be transversal to the tangent spaces in question.

\section{REFERENCES}

1. S. Abhyankar, Inversion and invariance of characteristic pairs, Amer. J. Math. 59 (1967), 363-372.

2. A. Azevedo, The jacobian ideal of a plane algebroid curve, Ph.D. Thesis, Purdue University, 1967.

3. J. Becker and J. Polking, $C^{k}$, weakly holomorphic functions on an analytic curve, Rice Univ. Stud. 59 (1972), Vol. 1-9, Proc. Conf. on Complex Analysis.

4. J. Becker and J. Stutz, The $C^{1}$ embedding dimension of analytic sets, Duke Math. J. 40 (1973), 221-231.

5. J. Becker, Holomorphic and differentiable tangent spaces to a complex analytic variety, J. Differential Geom. 12 (1977), 377-401.

6. T. Bloom, $C^{1}$ functions on an analytic variety, Duke Math. J. 36 (1969), 283-296.

7. Sherwood Ebey, The classification of singular points of algebraic curves, Trans. Amer. Math. Soc. 118 (1965), 454-471.

8. B. Malgrange, Ideals of differentiable functions, Tata Inst. Fund. Res., Bombay; Oxford Univ. Press, London, 1966.

9. R. Narasimhan, Introduction to analytic spaces, Lecture Notes in Math., vol. 25, Springer-Verlag, Berlin and New York, 1966.

10. F. Pham and B. Teissier, Fractions Lipschitziennes d'une algebra analytique complexe et saturation de Zariski.

11. K. Spallek, Differenzierbare und holomorphic Funktionen auf analytischen Mengen, Math. Ann. 161 (1965), 143-162.

12. __ Über Singularitateis analytisher Mengen, Math. Ann. 172 (1967), 249-268.

13. J. Stutz, Analytic sets as branched covering, Trans. Amer. Math. Soc. 166 (1972), 241-259.

14. B. Teissier and O. Zariski, Le probleme des modules pour les branches planes, Cours donne au Centre de Mathematiques de l'Ecole Polytechnique.

15. H. Whitney, Analytic extensions of differentiable functions defined in closed sets, Trans. Amer. Math. Soc. 36 (1936), 63-89.

16. __ Tangents to an analytic variety, Ann. of Math. (2) 78 (1963), 455-467. 
17. __ Local properties of analytic varieties, Differential and Combinatorial Topology, Princeton Univ. Press, Princeton, N. J., 1965.

18. E. Yoshimaga and T. Suzuki, The conductor number and weakly holomorphic functions on an analytic curve in $C^{2}$, Sci. Rep. Tokyo Kyoiku Diagaku Sect. A 13 (1975).

19. O. Zariski, Studies in equisingularity. I, II, III, Amer. J. Math. 87 (1965), 507-536; ibid. 87 (1965), 972-1006; ibid. 90 (1968), 961-1023.

20. __ Characterization of plane algebroid curves whose module of differentials has maximum torsion, Proc. Nat. Acad. U.S.A. Sci. 56 (1966), 781-786.

21. __ Open questions on the theory of equisingularity, Bull. Amer. Math. Soc. 77 (1971), 481-491.

Department of Mathematics, Brandeis University, Waltham, Massachusetts 02154 